OPEN ACCESS

Edited by:

Hans Ulrich Häring,

Tübingen University Hospital,

Germany

Reviewed by:

Abdurezak Ahmed Abdela,

Addis Ababa University, Ethiopia

Mohd Ashraf Ganie,

Sher-I-Kashmir Institute

of Medical Sciences, India

*Correspondence:

Anath Shalev

shalev@uab.edu

Specialty section:

This article was submitted to

Clinical Diabetes,

a section of the journal

Frontiers in Endocrinology

Received: 30 August 2020 Accepted: 07 December 2020

Published: 13 January 2021

Citation:

Crouse AB, Grimes T, Li P, Might M,

Ovalle $F$ and Shalev A (2021)

Metformin Use Is Associated

With Reduced Mortality in

a Diverse Population With

COVID-19 and Diabetes.

Front. Endocrinol. 11:600439.

doi: 10.3389/fendo.2020.600439

\section{Metformin Use Is Associated With Reduced Mortality in a Diverse Population With COVID-19 and Diabetes}

\author{
Andrew B. Crouse ${ }^{1}$, Tiffany Grimes ${ }^{2}$, Peng $L_{i}{ }^{3}$, Matthew Might ${ }^{1}$, Fernando Ovalle ${ }^{2}$ \\ and Anath Shalev ${ }^{2 *}$ \\ ${ }^{1}$ Hugh Kaul Precision Medicine Institute, University of Alabama at Birmingham, Birmingham, AL, United States, \\ ${ }^{2}$ Comprehensive Diabetes Center, Department of Medicine, Division of Endocrinology, Diabetes and Metabolism, University \\ of Alabama at Birmingham, Birmingham, AL, United States, ${ }^{3}$ School of Nursing, University of Alabama at Birmingham, \\ Birmingham, AL, United States
}

Background: Coronavirus disease-2019 (COVID-19) is a growing pandemic with an increasing death toll that has been linked to various comorbidities as well as racial disparity. However, the specific characteristics of these at-risk populations are still not known and approaches to lower mortality are lacking.

Methods: We conducted a retrospective electronic health record data analysis of 25,326 subjects tested for COVID-19 between 2/25/20 and 6/22/20 at the University of Alabama at Birmingham Hospital, a tertiary health care center in the racially diverse Southern U.S. The primary outcome was mortality in COVID-19-positive subjects and the association with subject characteristics and comorbidities was analyzed using simple and multiple linear logistic regression.

Results: The odds ratio of contracting COVID-19 was disproportionately high in Blacks/ African-Americans (OR 2.6; 95\% Cl 2.19-3.10; $\mathrm{p}<0.0001)$ and in subjects with obesity (OR 1.93; 95\% Cl 1.64-2.28; $\mathrm{p}<0.0001$ ), hypertension (OR 2.46; 95\% Cl 2.07-2.93; $\mathrm{p}<0.0001$ ), and diabetes (OR 2.11; 95\% Cl 1.78-2.48; $\mathrm{p}<0.0001$ ). Diabetes was also associated with a dramatic increase in mortality (OR 3.62; 95\% Cl 2.11-6.2; $p<0.0001$ ) and emerged as an independent risk factor in this diverse population even after correcting for age, race, sex, obesity, and hypertension. Interestingly, we found that metformin treatment prior to diagnosis of COVID-19 was independently associated with a significant reduction in mortality in subjects with diabetes and COVID-19 (OR 0.33; 95\% Cl 0.13$0.84 ; p=0.0210)$.

Conclusion: Thus, these results suggest that while diabetes is an independent risk factor for COVID-19-related mortality, this risk is dramatically reduced in subjects taking metformin prior to diagnosis of COVID-19, raising the possibility that metformin may provide a protective approach in this high risk population.

Keywords: African-American, coronavirus disease-2019, diabetes, metformin, mortality 


\section{INTRODUCTION}

Coronavirus disease-2019 (COVID-19) caused by the severe acute respiratory syndrome-coronavirus-2 (SARS-CoV-2) is a growing global pandemic that has devastated Asia, Europe, and now the United States. Its increasing death toll has been linked to higher age and a number of comorbidities including hypertension, obesity, and diabetes $(1,2)$, but approaches to counteract this trend are still lacking. Being a new disease, the specific patient characteristics of these at risk populations are also only starting to emerge with studies reported from China (3-5), Europe $(6,7)$ and more recently New York $(1,2)$.

However, currently still very little is known about patient characteristics in the U.S., particularly in more diverse communities with a large proportion of Blacks/AfricanAmericans such as in the South. This information is especially relevant as African-Americans have been disproportionally affected by this pandemic across the nation (8-10) and the prevalence of comorbidities including diabetes is very high in these communities (11). We therefore conducted a retrospective observational study of subjects diagnosed with COVID-19 at the University of Alabama at Birmingham (UAB) Hospital, a tertiary health care center in the South, aimed at identifying the patient characteristics and factors affecting mortality especially in the context of diabetes in this diverse cohort.

\section{METHODS}

\section{Study Design and Participants}

We conducted a retrospective analysis of de-identified electronic health record data (EHR). The sampling method consisted of including subjects consecutively tested for COVID-19 between February 25, 2020 and June 22, 2020 at UAB (Institutional Review Board protocol E160105006). To make the results as generalizable as possible and minimize any selection bias, completed testing within that time frame was also the only inclusion criteria and lack of outcome data in terms of survival was the only exclusion criteria. Subjects were categorized as confirmed COVID-19 positive or negative based on RT-PCR results from SARS-CoV-2 viral nucleic acid testing in respiratory specimens. The primary outcome was mortality and the effects of patient characteristics and comorbidities as documented in the EHR data (including 12 months before COVID-19 diagnosis) were analyzed. EHR data definitions for obesity included a body mass index (BMI) of $\geq 30 \mathrm{~kg} / \mathrm{m}^{2}$ and for hypertension a systolic blood pressure of $\geq 140 \mathrm{mmHg}$ and/ or a diastolic blood pressure of $\geq 90 \mathrm{mmHg}$. HbAlC was analyzed as a continuous variable. In terms of treatment, we focused on metformin and insulin as they were the two most common used drugs for diabetes and reliable electronic health record data were available. The number of subjects on other antidiabetic medications such as sodium-glucose cotransporter 2 (SGLT2) inhibitors or dipeptidyl peptidase IV (DPPIV) inhibitors was too small to allow for meaningful statistical analysis. This may have been due to the much higher costs of these newer medications and our cohort that included underserved communities.

\section{Statistical Analysis}

Patient characteristics and comorbidities were summarized as mean and standard deviation (SD) for continuous variables and frequency and proportion for categorical variables. In analysis, age was categorized into three groups: $<50,50-70$, and $>70$ years old. The association with COVID-19 diagnosis was explored utilizing a simple linear logistic regression for each of the potential risk factors and the raw odds ratio (OR) and the $95 \%$ confidence interval $(95 \% \mathrm{CI})$ were calculated for the strength of association. The associations with COVID-19 mortality for the potential risk factors were explored with both simple linear logistic regression for raw ORs and multiple linear logistic regression for adjusted ORs. Potential interactions were evaluated and removed from the multiple logistic regression model if not significant. The sample size of this study was determined by the available and eligible cases from EHR between February 25, 2020 and June 22, 2020 at UAB, including 24,722 COVID-19 negative and 604 COVID-19 positive subjects. This large sample size achieved $>80 \%$ power to detect even a very small effect (e.g., $\mathrm{OR}=1.25$ ) in the association of potential risk factors and contracting COVID19. Among COVID-19 positive subjects 67 were identified as deceased during the study period and this sample size achieved $>80 \%$ power to detect a medium effect size (e.g., $\mathrm{OR}=2.4$ or smaller depending on the distribution of risk factors) for the association of subject characteristics and mortality. The power analyses were conducted with a two-sided test in a logistic regression under the significance level of 0.05, using PASS 14 Power Analysis and Sample Size Software (NCSS, LLC. Kaysville, Utah). The statistical analyses were conducted using SAS 9.4 (Cary, NC).

\section{RESULTS}

\section{Subject Characteristics and Coronavirus 2019 Diagnosis}

The characteristics of the 24,722 subjects who tested negative for COVID-19 and 604 subjects who had a confirmed positive COVID-19 test are listed in Table 1. This low positivity rate of $2.4 \%$ is most likely due to the fact that asymptomatic hospital staff and patients coming for elective procedures were included in this screening. To explore the association between COVID-19 diagnosis and potential risk factors, a simple logistic regression was used. Notably, despite only representing $26 \%$ of the population in Alabama, the number of African-Americans who tested positive for COVID-19 was disproportionally high as African-Americans represented $52 \%$ of those who tested positive while accounting for only $30 \%$ of those who tested negative. This resulted in a highly significant odds ratio (OR 2.6, 95\% CI 2.19-3.10; $\mathrm{p}<0.0001$ ) (Table 1). In contrast, only $36 \%$ of COVID-19 positive subjects were Whites, whereas Whites made 
TABLE 1 | Subject characteristics and COVID-19 diagnosis.

\begin{tabular}{|c|c|c|c|c|c|}
\hline \multirow[t]{2}{*}{ Subject characteristics } & \multicolumn{2}{|c|}{ Covid-19 } & \multirow[t]{2}{*}{ Comparison } & \multirow[t]{2}{*}{ OR $(95 \% \mathrm{Cl})$} & \multirow[t]{2}{*}{ P-value } \\
\hline & Negative $(n=24,722)$ & Positive(n = 604) & & & \\
\hline \multicolumn{6}{|l|}{ Age Group } \\
\hline$<50$ years & 10626 (43.0\%) & 239 (39.6\%) & & & \\
\hline 50-70 years & 9862 (39.9\%) & 245 (40.6\%) & $50-70$ vs $<50$ & $1.10(0.92,1.32)$ & 0.2798 \\
\hline$>70$ years & 4234 (17.1\%) & 120 (19.9\%) & $>70$ vs $50-70$ & $1.14(0.91,1.42)$ & 0.2432 \\
\hline \multicolumn{6}{|l|}{ Race } \\
\hline African-American (AA) & 7498 (30.3\%) & 311 (51.5\%) & AA vs White & $2.61(2.19,3.10)$ & $<0.0001$ \\
\hline White & $13821(55.9 \%)$ & 220 (36.4\%) & & & \\
\hline Other & 3403 (13.8\%) & 73 (12.1\%) & & & \\
\hline \multicolumn{6}{|l|}{ Sex } \\
\hline Male & $10671(43.2 \%)$ & 272 (45.0\%) & $M$ vs $F$ & $1.06(0.90,1.25)$ & 0.4629 \\
\hline Female & 13841 (56.0\%) & 332 (55.0\%) & & & \\
\hline Unidentified & $210(0.8 \%)$ & & & & \\
\hline \multicolumn{6}{|l|}{ Obesity } \\
\hline Yes & 11167 (45.2\%) & 371 (61.4\%) & Y vs $N$ & $1.93(1.64,2.28)$ & $<0.0001$ \\
\hline No & 13555 (54.8\%) & 233 (38.6\%) & & & \\
\hline \multicolumn{6}{|l|}{ Hypertension } \\
\hline Yes & 11891 (48.1\%) & 420 (69.5\%) & Y vs N & $2.46(2.07,2.93)$ & $<0.0001$ \\
\hline No & 12831 (51.9\%) & $184(30.5 \%)$ & & & \\
\hline \multicolumn{6}{|l|}{ Diabetes } \\
\hline Yes & 5865 (23.7\%) & 239 (39.6\%) & Y vs N & $2.11(1.78,2.48)$ & $<0.0001$ \\
\hline No & 18857 (76.3\%) & 365 (60.4\%) & & & \\
\hline
\end{tabular}

up $56 \%$ of those who tested negative, further underlining the racial disparity. Interestingly, $70 \%$ of all subjects diagnosed with COVID-19 had pre-existing hypertension, 61\% had obesity and $40 \%$ had diabetes and the risk of being diagnosed with COVID19 while suffering from any one of these comorbidities was significantly elevated $(\mathrm{p}<0.0001)$ (Table 1$)$. In the case of diabetes, $92 \%$ of subjects also had hypertension and $74 \%$ were obese, which may have further contributed to the increased risk observed in this population. Overall, these results are very much in line with global observations and suggested that our cohort provided a representative sample.

\section{Characteristics and Mortality of Coronavirus 2019 Positive Subjects}

Overall mortality in COVID-19 positive individuals was $11 \%$, but varied a lot depending on a number of subject characteristics. Ninety three percent of deaths occurred in subjects over the age of 50 and male sex as well as hypertension were associated with a

TABLE 2 | Characteristics and mortality of COVID-19 positive subjects.

\begin{tabular}{|c|c|c|c|c|c|}
\hline Subject characteristics & \multicolumn{2}{|c|}{ Mortality } & Comparison & OR $(95 \% \mathrm{Cl})$ & P-value \\
\hline \multicolumn{6}{|l|}{ Age Group } \\
\hline 50-70 years & $216(40.2 \%)$ & $29(43.3 \%)$ & $50-70$ vs $<50$ & $6.28(2.39,16.5)$ & 0.0002 \\
\hline$>70$ years & 87 (16.2\%) & $33(49.2 \%)$ & $>70$ vs $50-70$ & $2.83(1.62,4.93)$ & 0.0003 \\
\hline \multicolumn{6}{|l|}{ Race } \\
\hline Other & $68(12.7 \%)$ & $5(7.5 \%)$ & & & \\
\hline \multicolumn{6}{|l|}{ Sex } \\
\hline Male & $231(43.0 \%)$ & $41(61.2 \%)$ & M vs F & $1.52(1.19,1.72)$ & 0.0055 \\
\hline Female & $306(57.0 \%)$ & $26(38.8 \%)$ & & & \\
\hline \multicolumn{6}{|l|}{ Obesity } \\
\hline Yes & 328 (61.1\%) & $43(64.2 \%)$ & Y vs N & $1.14(0.67,1.94)$ & 0.6234 \\
\hline \multicolumn{6}{|l|}{ Diabetes } \\
\hline Yes & 194 (36.1\%) & 45 (67.2\%) & Y vs N & $3.62(2.11,6.20)$ & $<0.0001$ \\
\hline No & 343 (63.9\%) & 22 (32.8\%) & & & \\
\hline
\end{tabular}


significantly elevated risk of death as assessed by bivariate logistic regression analysis (Table 2). In addition, diabetes was associated with a dramatic increase in mortality (OR 3.62; 95\% CI 2.11-6.2; $\mathrm{p}<0.0001$ ). In fact, $67 \%$ of deaths occurred in subjects with diabetes.

We also conducted multiple logistic regression analysis with age, race, sex, obese status, hypertension status, and diabetes status as covariates and the adjusted odds ratios and 95\% CIs are illustrated in Figure 1. Specifically, after controlling for these other covariates, age, sex, and diabetes emerged as the major factors significantly associated with COVID-19 related mortality, suggesting that they are independent risk factors.

\section{Characteristics and Mortality of Coronavirus 2019 Positive Subjects With Diabetes}

Based on the identification of diabetes as an independent risk factor for mortality in COVID-19 positive subjects, we explored potential additional risk factors within this diabetic subgroup. Notably, higher

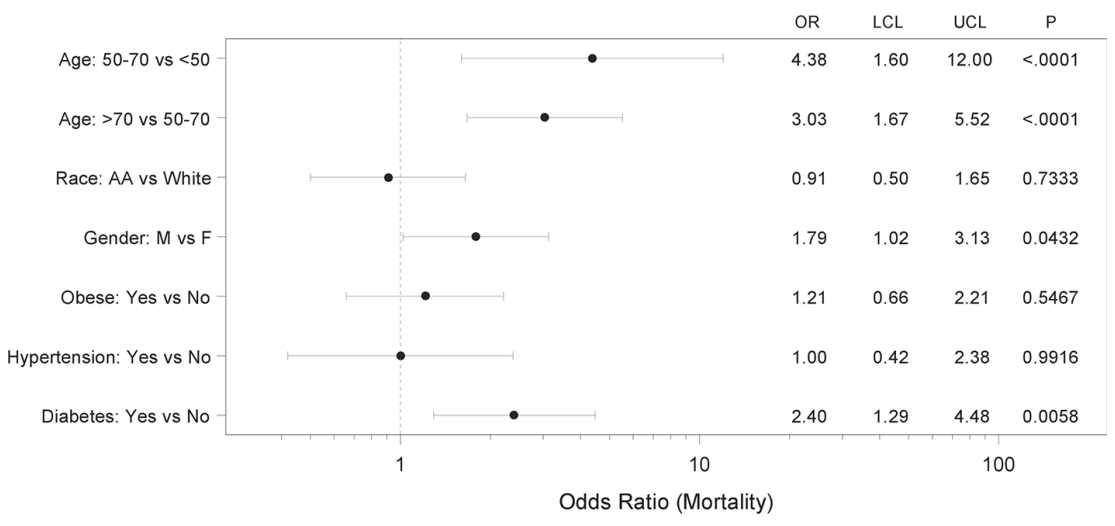

FIGURE 1 | Forest plot showing adjusted mortality risk in subjects with coronavirus 2019 (COVID-19). Multiple logistic regression analysis with age, race, sex, obese status, hypertension status, and diabetes status as covariates was performed. The regression yielded a significant model $(\mathrm{p}<0.0001)$ with $\mathrm{AUC}$ of $0.79(95 \% \mathrm{Cl}$ $0.74-0.85$ ) and the adjusted odds ratios (OR), 95\% confidence intervals (LCL-UCL) and corresponding P-values are shown.

TABLE 3 | Characteristics and mortality of COVID-19 positive subjects with diabetes.

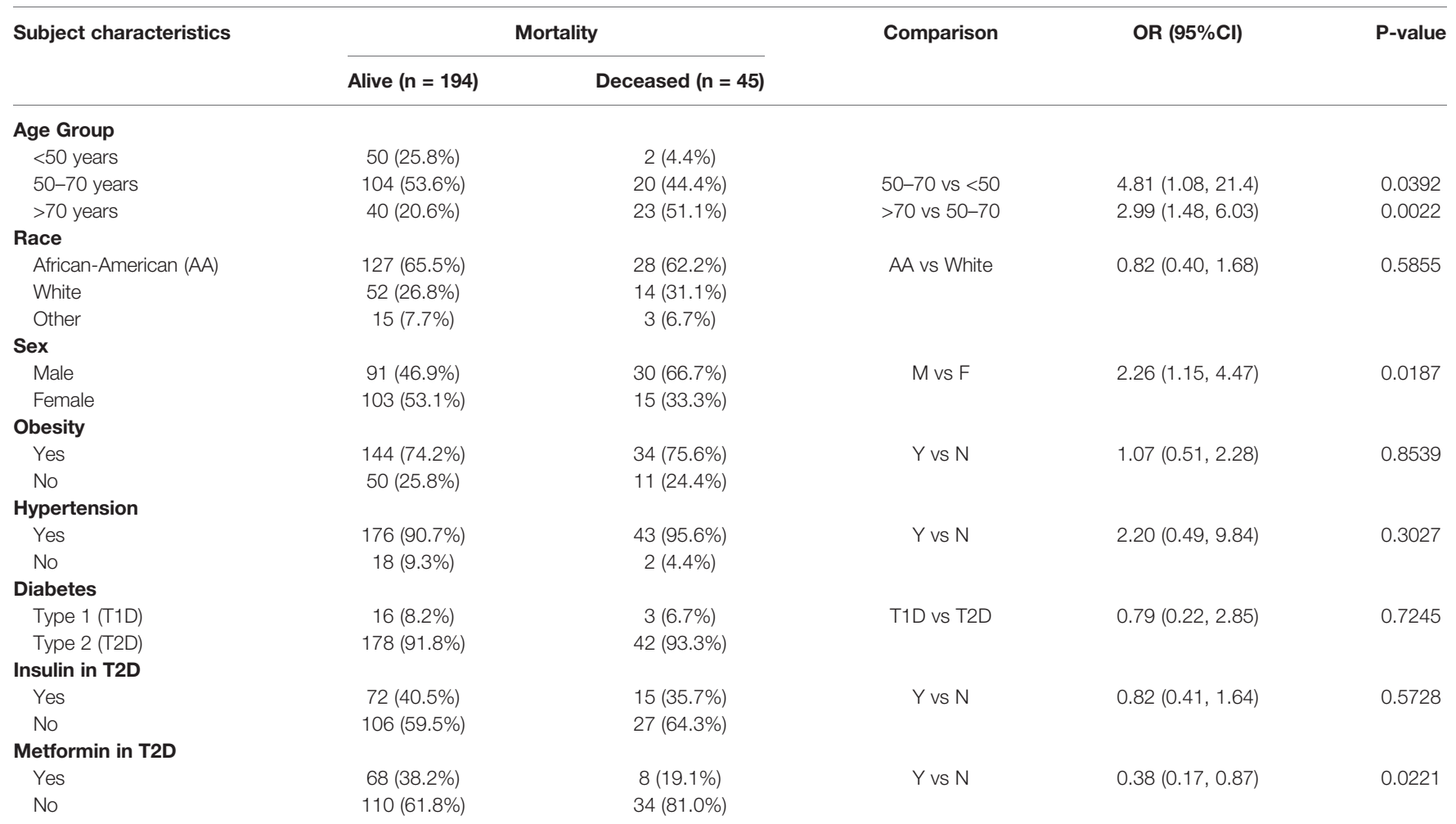


age and male sex continued to be associated with increased mortality in the context of diabetes, while no significant difference between type 1 (T1D) and type 2 diabetes (T2D) was observed (Table 3). Next, we investigated the effects of diabetes treatment on adverse COVID-19 outcome. We focused on insulin and metformin as the two most common medications prescribed for T2D. To avoid confounding effects from insulin being initiated for stress hyperglycemia and from metformin being discontinued in hospitalized patients, only medications used prior to the diagnosis of COVID-19 were considered. Interestingly, while prior insulin use did not seem to affect mortality risk, metformin use significantly reduced the odds of dying (OR 0.38; 95\% CI 0.17-0.87; $\mathrm{p}=0.0221$ ). In fact, with $11 \%$ the mortality of metformin users was comparable to that of the general COVID-19-positive population and dramatically lower than the $24 \%$ mortality observed in subjects with diabetes and not on metformin. Of note, this beneficial effect of metformin use on adverse outcome remained even when subjects with chronic kidney disease or chronic heart failure, classical contraindications for metformin, were excluded from the analysis (OR 0.17; 95\% CI 0.04-0.79; $\mathrm{p}=0.0231$ ). This makes any potential confounding effects from skewing metformin users toward healthier subjects without these additional comorbidities, very unlikely. To further determine whether the effect might be just driven by female sex, as one report proposed that women particularly benefit from metformin (12), we also analyzed males separately. Interestingly, the odds ratio of dying remained significantly lower in male subjects on metformin (OR 0.28; 95\% CI 0.09-0.88; p=0.0286).

Moreover, we again performed multiple logistic regression analysis with metformin use, insulin use, age, race, sex, obese status, and hypertension status as covariates and the adjusted odds ratios and 95\% CIs are shown in Figure 2. Specifically, after controlling for other covariates, age, sex, and metformin use emerged as independent factors affecting COVID-19 related mortality. Interestingly, even after controlling for all these other covariates, the likelihood of death for subjects taking metformin for their T2D was significantly less than for those who did not take metformin (OR 0.33 ; 95\% CI $0.13-0.84$ $\mathrm{p}=0.0210$ ). In this regard it is also important to note that subjects not taking metformin did not have more severe metabolic disease or diabetes than those on metformin as demonstrated by comparable or even lower body mass index (BMI) and hemoglobin A1C (HbA1C) values (Table 4).

Still, since metformin is known and used for its weight neutral or even weight lowering properties, while improving glycemic

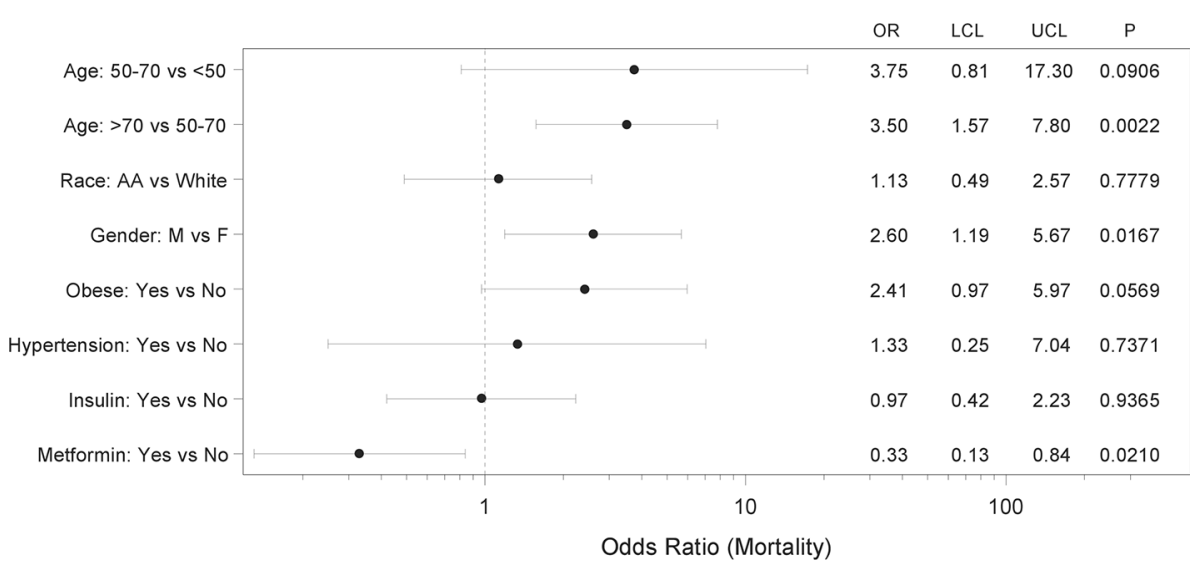

FIGURE 2 | Forest plot showing adjusted mortality risk in subjects with coronavirus 2019 (COVID-19) and T2D. Multiple logistic regression analysis with metformin use, insulin use, age, race, sex, obese status, and hypertension status as covariates was performed and yielded a significant model ( $p=0.0001)$ with AUC of 0.77 $(0.69,0.85)$. The adjusted odds ratios (OR), 95\% confidence intervals (LCL-UCL), and corresponding P-values are shown.

TABLE 4 | BMI, BG, and HbA1C of subjects with COVID-19 and T2D treated with/without metformin.

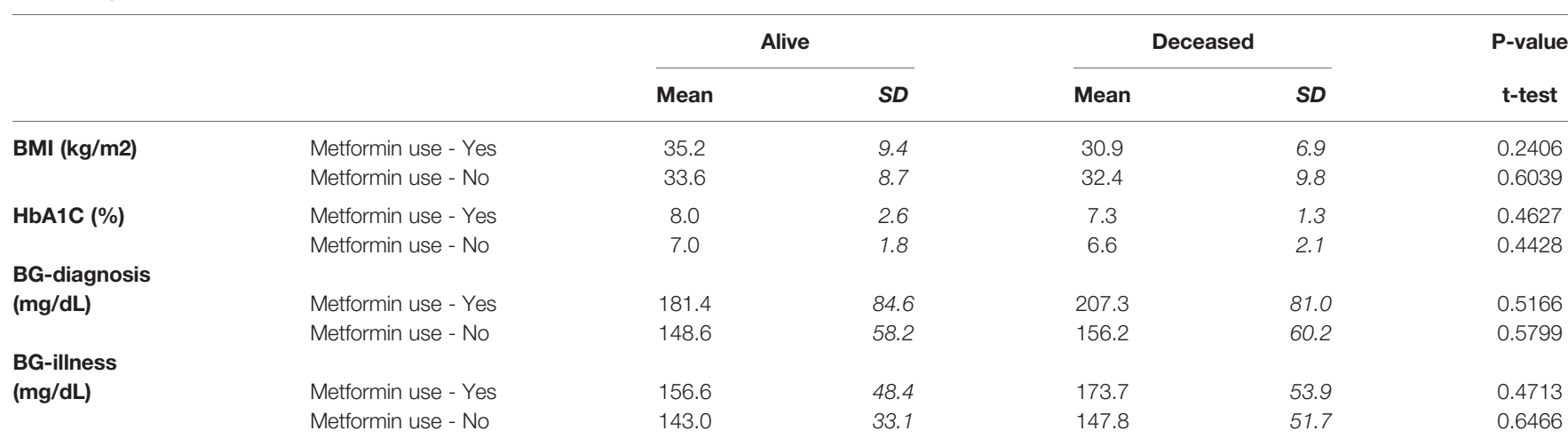


control in T2D (13), we wondered whether these effects might contribute to the reduced risk of COVID-19 related mortality. However, neither BMI nor HbA1C were lower in metformin users who survived as compared to those who died (Table 4). While surprising, this is consistent with the notion that longterm glycemic control does not affect COVID-19 outcome, as recently reported (6). Also, only one subject in our cohort experienced a hyperosmolar hyperglycemic state and ended up surviving and there were no subjects with diabetic ketoacidosis (DKA). Moreover, blood glucose at diagnosis and during the illness were not significantly different in metformin-users who survived as compared to those who died (Table 4). This further suggests that other factors may play a more important role in terms of the metformin effects on outcome in the context of COVID-19 and T2D.

\section{DISCUSSION}

In summary, the findings of this study in a racially diverse population demonstrate that diabetes is an independent risk factor associated with increased mortality in individuals with COVID-19, whereas metformin treatment is associated with dramatically reduced mortality in subjects with T2D even after correcting for multiple covariates.

Most strikingly, we found that metformin use prior to the diagnosis of COVID-19 was associated with a $\sim 3$-fold decrease in mortality and significantly lower unadjusted and adjusted odds ratios in subjects with diabetes. Of note, this effect remained even after correcting for age, sex, race, obesity, and hypertension or chronic kidney disease and heart failure. Interestingly and in alignment with this finding, an early report from Wuhan, China also suggested that metformin was associated with decreased mortality in hospitalized COVID-19 patients with diabetes in (14). Metformin was also found to be associated with reduced risk of early death in the French CORONADO study (6) and most recently, it was suggested to be associated with decreased mortality in women with COVID-19 based on a UnitedHealth data analysis (12). The fact that such similar results were obtained in different populations from around the world suggests that the observed reduction in mortality risk, associated with metformin use in subjects with T2D and COVID-19, might be generalizable. In fact, a very recent metaanalysis of this collective work concluded that metformin has benefits in reducing the mortality rate from COVID-19 (15). Furthermore, these findings underline the importance of following general diabetes treatment and prevention guidelines and not delaying or discontinuing any metformin treatment. Especially during this pandemic that puts subjects with diabetes at particularly high risk, this treatment might not only help with diabetes management, but also reduce the risk of adverse outcome in case of a COVID-19 infection.

At this point, the mechanisms by which metformin might improve prognosis in the context of COVID-19 are not known. Our findings suggest that they go beyond any expected improvement in glycemic control or obesity as blood glucose,
$\mathrm{HbA1C}$, or BMI were not lower in COVID-19 survivors on metformin. Interestingly, metformin has previously been shown to also have anti-inflammatory $(16,17)$ and anti-thrombotic effects $(18,19)$ and excessive inflammatory responses, e.g., cytokine storm as well as disseminated thromboembolic events have been recognized as deadly complications of COVID-19 infection (20-22). It is therefore tempting to speculate that by exerting some of its anti-fibrinolytic activities (18) and inhibiting inflammatory cytokines such as tumor necrosis factor alpha or interleukin-6 $(16,17)$, suspected to play a role in the immune response to COVID-19 (12), metformin might improve outcome. In fact, even prior to the COVID-19 pandemic, preadmission metformin use was found to be associated with reduced mortality in medical and surgical intensive care patients with T2D (23).

While diabetes has been recognized universally as one of the major comorbidities adversely affecting COVID-19 outcome, the factors responsible for this phenomenon are not well understood. Of note, we found that the increased mortality risk of subjects with diabetes persisted even after correcting for covariates such as age, race, obesity, and hypertension, suggesting that while these factors might contribute to a worse outcome, they cannot fully account for it. In the CORONADO study higher glucose levels at admission were associated with a trend toward increased mortality (6) and in-hospital hyperglycemia contributed to worse prognosis in a large multicenter study of patients with COVID19 from Wuhan (24). Consistently, we found in general slightly higher glucose levels in subjects who died. However, neither blood glucose levels at diagnosis nor during the illness were lower in metformin-users, making it very unlikely that better control of blood glucose was responsible for the improved outcome observed in subjects taking metformin. Also, long-term glycemic control as assessed by HbA1C did not affect mortality in our study, in alignment with previous reports (6). Similar to the issue with metformin, other factors such as diabetesassociated inflammation (25) and coagulopathy (26) may therefore play a more prominent role in this regard. In addition, a recent report also demonstrated that pancreatic beta cells can get infected and damaged by SARS-CoV-2 (27) providing a potential explanation for the extremely high insulin requirements seen in some subject with COVID-19 as well as the development of diabetic ketoacidosis and possibly new onset diabetes $(28,29)$.

Higher age and male sex were the other independent risk factors associated with increased mortality that we found consistently across subjects with and without diabetes. In fact, the mortality rate in males was more than two-fold higher than in females, which is in line with previous studies (30). Many theories have been proposed for why this might be, including the different concentrations of sex steroids, different fat distribution, different level of circulating pro-inflammatory cytokines, and different innate and adaptive immune response to viral infections $(30,31)$. In fact, due to this striking sexual dimorphism, studies using antiandrogens in COVID-19 positive men are currently ongoing. In any case, it is encouraging that the beneficial effects of metformin remained strong in the male subjects of our study. 
In our cohort being African-American appeared to be primarily a risk factor for contracting COVID-19 rather than for mortality. These findings are supported by a recent study using an integrated-delivery health system cohort with similar demographics ( $30 \%$ Blacks/African-American), which found that Black race was not associated with higher in-hospital mortality than White race. This suggests that any racial disparity observed may be more likely due to exposure risk and external, socioeconomic factors than to biological differences. The fact that other geographic areas (mostly with a smaller proportion of African-Americans), did see a difference in mortality (10), might be related to issues with healthcare access.

Limitations of the study include the size that did not allow for any separate analyses of additional subgroups such as T1D or subjects on other anti-diabetic drugs besides metformin. On the other hand, the diverse community comprising a large proportion of African-American men and women represents a unique feature of our study. Also, the fact that in our study metformin-users did not have lower blood glucose levels than non-users, suggested that better metabolic control was unlikely to be responsible for the improved outcome observed in these subjects.

Taken together, our study reaffirmed the role of the major comorbidities associated with COVID-19 in a more diverse population with a higher proportion of African-Americans, demonstrated the prominence of diabetes as an independent risk factor associated with higher mortality and revealed that metformin use prior to a diagnosis of COVID-19 was associated with a consistent and robust decrease in mortality in subjects with diabetes. Future studies will have to explore how metformin might confer these protective effects, provide a careful risk benefit assessment and determine whether the indications for metformin treatment should be broadened in the face of the ongoing COVID-19 pandemic.

\section{REFERENCES}

1. Richardson S, Hirsch JS, Narasimhan M, Crawford JM, McGinn T, Davidson KW, et al. Presenting Characteristics, Comorbidities, and Outcomes Among 5700 Patients Hospitalized With COVID-19 in the New York City Area. Jama (2020) 323(20):2052-9. doi: 10.1001/jama.2020.6775

2. Palaiodimos L, Kokkinidis DG, Li W, Karamanis D, Ognibene J, Arora S, et al. Severe obesity, increasing age and male sex are independently associated with worse in-hospital outcomes, and higher in-hospital mortality, in a cohort of patients with COVID-19 in the Bronx, New York. Metabol: Clin Experiment (2020) 108:154262. doi: 10.1016/j.metabol.2020.154262

3. Cai Q, Chen F, Wang T, Luo F, Liu X, Wu Q, et al. Obesity and COVID-19 Severity in a Designated Hospital in Shenzhen, China. Diabetes Care (2020) 43(7):1392-8. doi: 10.2337/dc20-0576

4. Chen Q, Zheng Z, Zhang C, Zhang X, Wu H, Wang J, et al. Clinical characteristics of 145 patients with corona virus disease 2019 (COVID-19) in Taizhou, Zhejiang, China. Infection (2020) 48(4):543-51. doi: 10.1007/ s15010-020-01432-5

5. Wang D, Hu B, Hu C, Zhu F, Liu X, Zhang J, et al. Clinical Characteristics of 138 Hospitalized Patients With 2019 Novel Coronavirus-Infected Pneumonia in Wuhan, China. Jama (2020) 323(11):1061-9. doi: 10.1001/jama.2020.1585

6. Cariou B, Hadjadj S, Wargny M, Pichelin M, Al-Salameh A, Allix I, et al. Phenotypic characteristics and prognosis of inpatients with COVID-19 and diabetes: the CORONADO study. Diabetologia (2020) 63(8):1500-15. doi: 10.1007/s00125-020-05180-X

\section{DATA AVAILABILITY STATEMENT}

The original contributions presented in the study are included in the article/supplementary material. Further inquiries can be directed to the corresponding author.

\section{ETHICS STATEMENT}

The studies involving human participants were reviewed and approved by UAB Institutional Review Board. Written informed consent for participation was not required for this study in accordance with the national legislation and the institutional requirements.

\section{AUTHOR CONTRIBUTIONS}

AC and TG were responsible for data acquisition and analysis. PL performed all the statistical analyses. MM and FO helped with the approach and interpretation. AS conceived the study and wrote the manuscript. All authors contributed to the article and approved the submitted version.

\section{ACKNOWLEDGMENTS}

AS is supported by National Institutes of Health grants R01DK078752 and U01DK120379 and the UAB Center for Clinical and Translational Science (CCTS) by UL1TR001417. This manuscript has been released as a pre-print at medRxiv https://www.medrxiv.org/content/10.1101/2020.07.29.2016 4020v1 (32).

7. Scheen AJ, Marre M, Thivolet C. Prognostic factors in patients with diabetes hospitalized for COVID-19: Findings from the CORONADO study and other recent reports. Diabetes Metab (2020) 46(4):265-71. doi: 10.1016/ j.diabet.2020.05.008

8. Price-Haywood EG, Burton J, Fort D, Seoane L. Hospitalization and Mortality among Black Patients and White Patients with Covid-19. New Engl J Med (2020) 382(26):2534-43. doi: 10.1056/NEJMsa2011686

9. Tai DBG, Shah A, Doubeni CA, Sia IG, Wieland ML. The Disproportionate Impact of COVID-19 on Racial and Ethnic Minorities in the United States. Clin Infect Dis Off Publ Infect Dis Soc A (2020). doi: 10.1093/cid/ciaa815

10. Holmes L Jr, Enwere M, Williams J, Ogundele B, Chavan P, Piccoli T, et al. Black-White Risk Differentials in COVID-19 (SARS-COV2) Transmission, Mortality and Case Fatality in the United States: Translational Epidemiologic Perspective and Challenges. Int J Environ Res Public Health (2020) 17 (12):4322. doi: 10.3390/ijerph17124322

11. Golden SH, Yajnik C, Phatak S, Hanson RL, Knowler WC. Racial/ethnic differences in the burden of type 2 diabetes over the life course: a focus on the USA and India. Diabetologia (2019) 62(10):1751-60. doi: 10.1007/s00125-019-4968-0

12. Bramante $\mathrm{C}$, Ingraham N, Murray $\mathrm{T}$, Marmor S, Hoversten S, Gronski J, et al. Observational Study of Metformin and Risk of Mortality in Patients Hospitalized with Covid-19. MedRxiv Prepr Server Health Sci (2020). doi: 10.1101/2020.06.19.20135095

13. Tan MH, Alquraini H, Mizokami-Stout K, MacEachern M. Metformin: From Research to Clinical Practice. Endocrinol Metab Clinics North A (2016) 45 (4):819-43. doi: 10.1016/j.ecl.2016.06.008 
14. Luo P, Qiu L, Liu Y, Liu XL, Zheng JL, Xue HY, et al. Metformin Treatment Was Associated with Decreased Mortality in COVID-19 Patients with Diabetes in a Retrospective Analysis. Am J Trop Med Hygiene (2020) 103 (1):69-72. doi: 10.4269/ajtmh.20-0375

15. Hariyanto TI, Kurniawan A. Metformin use is associated with reduced mortality rate from coronavirus disease 2019 (COVID-19) infection. Obes Med (2020) 19:100290. doi: 10.1016/j.obmed.2020.100290

16. Cameron AR, Morrison VL, Levin D, Mohan M, Forteath C, Beall C, et al. Anti-Inflammatory Effects of Metformin Irrespective of Diabetes Status. Circ Res (2016) 119(5):652-65. doi: 10.1161/CIRCRESAHA.116.308445

17. Tsoyi K, Jang HJ, Nizamutdinova IT, Kim YM, Lee YS, Kim HJ, et al. Metformin inhibits HMGB1 release in LPS-treated RAW 264.7 cells and increases survival rate of endotoxaemic mice. Br J Pharmacol (2011) 162 (7):1498-508. doi: 10.1111/j.1476-5381.2010.01126.x

18. Grant PJ. The effects of metformin on the fibrinolytic system in diabetic and non-diabetic subjects. Diabete Metabol (1991) 17(1 Pt 2):168-73.

19. Xin G, Wei Z, Ji C, Zheng H, Gu J, Ma L, et al. Metformin Uniquely Prevents Thrombosis by Inhibiting Platelet Activation and mtDNA Release. Sci Rep (2016) 6:36222. doi: 10.1038/srep36222

20. McFadyen JD, Stevens H, Peter K. The Emerging Threat of (Micro) Thrombosis in COVID-19 and Its Therapeutic Implications. Circ Res (2020) 127(4):571-87. doi: 10.1161/CIRCRESAHA.120.317447

21. Connors JM, Levy JH. COVID-19 and its implications for thrombosis and anticoagulation. Blood (2020) 135(23):2033-40. doi: 10.1182/ blood.2020006000

22. Khan IH, Savarimuthu S, Leung MST, Harky A. The need to manage the risk of thromboembolism in COVID-19 patients. J Vasc Surg (2020) 72(3):799804. doi: 10.1016/j.jvs.2020.05.015

23. Christiansen C, Johansen M, Christensen S, O'Brien JM, Tonnesen E, Sorensen H. Preadmission metformin use and mortality among intensive care patients with diabetes: a cohort study. Crit Care (2013) 17(5):R192. doi: $10.1186 / \mathrm{cc} 12886$

24. Zhu L, She ZG, Cheng X, Qin JJ, Zhang XJ, Cai J, et al. Association of Blood Glucose Control and Outcomes in Patients with COVID-19 and Pre-existing Type 2 Diabetes. Cell Metab (2020) 31(6):1068-77 e3. doi: 10.1016/ j.cmet.2020.04.021
25. Mugabo Y, Li L, Renier G. The connection between C-reactive protein (CRP) and diabetic vasculopathy. Focus on preclinical findings. Curr Diabetes Rev (2010) 6(1):27-34. doi: 10.2174/157339910790442628

26. Nogami K, Muraki I, Imano H, Iso H. Risk of disseminated intravascular coagulation in patients with type 2 diabetes mellitus: retrospective cohort study. BMJ Open (2017) 7(1):e013894. doi: 10.1136/bmjopen-2016-013894

27. Yang L, Han Y, Nilsson-Payant BE, Gupta V, Wang P, Duan X, et al. A Human Pluripotent Stem Cell-based Platform to Study SARS-CoV-2 Tropism and Model Virus Infection in Human Cells and Organoids. Cell Stem Cell (2020) 27(1):125-36 e7. doi: 10.1016/j.stem.2020.06.015

28. Apicella M, Campopiano MC, Mantuano M, Mazoni L, Coppelli A, Del Prato S. COVID-19 in people with diabetes: understanding the reasons for worse outcomes. Lancet Diabetes Endocrinol (2020) 8(9):782-92. doi: 10.1016/S2213-8587(20)30238-2

29. Li J, Wang X, Chen J, Zuo X, Zhang H, Deng A. COVID-19 infection may cause ketosis and ketoacidosis. Diabetes Obes Metab (2020) 22(10):1935-41. doi: $10.1111 /$ dom.14057

30. Mauvais-Jarvis F. Aging, Male Sex, Obesity, and Metabolic Inflammation Create the Perfect Storm for COVID-19. Diabetes (2020) 69(9):1857-63. doi: 10.2337/dbi19-0023

31. Klein SL, Flanagan KL. Sex differences in immune responses. Nat Rev Immunol (2016) 16(10):626-38. doi: 10.1038/nri.2016.90

32. Crouse A, Grimes T, Li P, Might M, Ovalle F, Shalev A. Metformin Use Is Associated with Reduced Mortality in a Diverse Population with Covid-19 and Diabetes. MedRxiv Prepr Server Health Sci (2020). doi: 10.1101/ 2020.07.29.20164020

Conflict of Interest: The authors declare that the research was conducted in the absence of any commercial or financial relationships that could be construed as a potential conflict of interest.

Copyright (C) 2021 Crouse, Grimes, Li, Might, Ovalle and Shalev. This is an openaccess article distributed under the terms of the Creative Commons Attribution License (CC BY). The use, distribution or reproduction in other forums is permitted, provided the original author(s) and the copyright owner(s) are credited and that the original publication in this journal is cited, in accordance with accepted academic practice. No use, distribution or reproduction is permitted which does not comply with these terms. 